\title{
Synergism Effect between Phenolic Metabolites and Endogenous Antioxidants in Terms of Antioxidant Activity
}

\author{
María Noguer, Ana B. Cerezo, M. Luisa Moyá, Ana M. Troncoso, \\ M. Carmen García-Parrilla* \\ Nutrición y Bromatología, Facultad de Farmacia, Universidad de Sevilla, Sevilla, Spain \\ Email: ${ }^{\text {mcparrilla@us.es }}$
}

Received 18 February 2014; revised 15 March 2014; accepted 22 March 2014

Copyright (C) 2014 by authors and Scientific Research Publishing Inc. This work is licensed under the Creative Commons Attribution International License (CC BY). http://creativecommons.org/licenses/by/4.0/

\section{Abstract}

Polyphenolic compounds, widely distributed in plant kingdom, have been exhaustively studied for their bioactive properties specially antioxidant activity. However, they are extensively metabolized by human organism and the resulting metabolites are largely responsible for their effects. Furthermore, they may interact with the endogenous antioxidant network being this possibility scarcely studied. Plasma antioxidant network encompasses antioxidant enzymes and other substances such as uric acid. In addition, ascorbic acid is the major compound representing water soluble compartment both in foods and human body. The interaction of this vitamin with phenolic compound is largely unexplored. This work aims to study if there is a synergic effect between phenolic metabolites and main antioxidants (uric and ascorbic acid). For this purpose, the antioxidant activity was evaluated in terms of ORAC (oxygen radical absorbance capacity) and FRAP (ferricreducing antioxidant power) as these tests involved either HAT (Hydrogen Atom Transfer) or SET (Single Electron Transfer) mechanisms. Additionally, a kinetic studied was developed to test if the rate constant presented a synergic effect. Protocatechuic acid, 3,4-dihydroxyphenylacetic acid, 3,4-dihydroxyphenylpropionic acid and 3-hydroxyphenylacetic acid were selected as they were metabolites of polyphenol compounds such as anthocyanins, quercetin, neohesperidin, chlorogenic acid and hesperetin present in wines, orange and strawberries. A synergic effect was proved for the combination of ascorbic acid with 3,4-dihydroxyphenylacetic acid, both in terms of antioxidant activity and potent increase of velocity of the antioxidant reaction that took place.

\section{Keywords}

Kinetics, Ascorbic Acid, Uric Acid, ORAC, FRAP

\footnotetext{
${ }^{*}$ Corresponding author.
}

How to cite this paper: Noguer, M., et al. (2014) Synergism Effect between Phenolic Metabolites and Endogenous Antioxidants in Terms of Antioxidant Activity. Advances in Chemical Engineering and Science, 4, $258-265$. 


\section{Introduction}

Phenolic compounds present in wines and in many other foods and beverages exhibit marked antioxidant activity and their in vitro antioxidant properties have been studied in depth [1]-[4]. Methods to assess the antioxidant activity are based on Hydrogen Atom Transfer (HAT) and others on Single Electron Transfer (SET). Both detect the ability of a potential antioxidant to either scavenge a radical or to transfer one electron to reduce any compound, including radicals. The end result is the same, regardless of mechanism, but kinetics and potential for side reactions differ [5]. Hence, ORAC (oxygen radical absorbance capacity) method is based on a hydrogen atom transfer (HAT) reaction mechanism and FRAP (ferric-reducing antioxidant power) assay is a SET-based method.

Moreover, the correlation between antioxidant activity and the phenolic composition has been considered by different authors for different foods and beverages, including wine and strawberries, among others [6]-[10], following that the different categories of phenolic compounds are contributing in a different extent to the final antioxidant capacity. Depending on the antioxidant assay employed this contribution can range between 12.8 - $20 \%$ and 32\% - 40\% for phenolic acids, and anthocyanins and flavanols, respectively [4].

At the human body level, the biological antioxidant defense encompasses an array of mechanisms including antioxidant enzymes (CAT, SOD, GPx,GR), endogenous antioxidants (glutation, uric acid, albumin) and dietary antioxidants (vitamins C, A, and E, Se, carotenoids and polyphenols) among other bioactive compounds [11].

However, after ingestion, dietary (poly)phenols appear in the circulatory system not as the parent compounds, but as phase II metabolites, and their presence in plasma after dietary intake rarely exceeds nM concentrations. Substantial quantities of both the parent compounds and their metabolites pass to the colon where they are degraded by the action of the local microbiota, giving rise principally to small phenolic acid and aromatic compounds that are absorbed into the circulatory system [12]. Several phenolic acids (protocatechuic acid, 3,4dihydroxyphenylacetic acid, 3,4-dihydroxyphenylpropionic acid and 3-hydroxyphenylacetic acid) are examples of these metabolites coming from dietary polypehnols.

On the other hand, antioxidant activity effect can be determined both by the total moles of radicals scavenged and by the velocity they react to remove the radical from the media. This is of particular biological significance since the faster the reaction takes places the lesser the biological targets are damaged. At the same time, at the biological level we have many antioxidants available, and as a result they may act synergistically.

The aim of this work is to evaluate the kinetics and synergism of different antioxidants (vitamin C, uric acid), and polyphenol metabolites (protocatechuic acid, 3,4-dihydroxyphenylacetic acid, 3,4-dihydroxy-phenylpropionic acid and 3-hydroxyphenylacetic acid) that are present in biological fluids as a consequence of the ingestion of polyphenol-rich foods.

\section{Material and Methods}

\subsection{Samples}

A total of 19 aqueous standards's solutions of different antioxidants (uric acid, ascorbic acid) and metabolites of phenolic compounds (protocatechuic acid, 3,4-dihydroxyphenylacetic acid, 3,4-dihydroxyphenylpropionic acid and 3-hydroxyphenylacetic acid) were prepared. The final concentrations assessed were $0.416 \mathrm{mM}$.

\subsection{Chemicals}

The chemicals and stardand compounds were purchased from Cayman Chemical Company [2,2'-azobis-(2amidinopropane)-dihydrochloride (AAPH)], Fluka [fluorescein], Sygma-Aldrich [ferric 2,4,6-tripyridyl-s-triazine (TPTZ), acetic acid and 6-hydroxy-2,5,7,8-tetramethylchroman-2-carboxylic acid (Trolox), uric acid, protocatechuic acid, 3,4-dihydroxyphenylacetic acid, 3,4-dihydroxyphenylpropionic and 3-hydroxyphenylacetic acid] and Merck [ascorbic acid].

\subsection{ORAC Method}

Fluorimetric measurements were recorded in F-2500 Fluorescence Spectrophotometer Hitachi and temperature control P-SelecterTectron Bio at $37^{\circ} \mathrm{C}$. A total of $150 \mu \mathrm{l}$ of solution problem, $150 \mu$ l fluorescein solution (2.934 $\mathrm{mg} / \mathrm{L})$ and $75 \mu \mathrm{AAPH}(221.25 \mathrm{mM})$ were mixed in a fluorimetric cuvette. Fluorescence was recorded for 60 min until the final value is less than $5 \%$ of the initial value (excitation wavelength is set at $490 \mathrm{~nm}$; emission 
wavelength is set at $515 \mathrm{~nm})$. Trolox solution $(20 \mu \mathrm{M})$ was used as standard. Phosphate buffer (PBS) (75 mM, $\mathrm{pH}$ 7) was used instead of the sample to assay the blank. A triplicated was performed in every case. Results are calculated as ORAC values using areas under the fluorescein decay curve between the blank and the sample and are expressed as Trolox Equivalents [4].

\subsection{FRAP Method}

This procedure is based on a Single Electron Transfer (SET) reaction mechanism, as follows:

$$
\mathrm{Fe}(\mathrm{TPTZ})_{2}^{3+}+\mathrm{ArOH} \rightarrow \mathrm{Fe}(\mathrm{TPTZ})_{2}^{2+}+\mathrm{ArOH}^{+}
$$

Equation (1) expresses the redox reaction when the ferric tripyridyltriazine complex $\left(\mathrm{Fe}(\mathrm{TPTZ})_{2}^{3+}\right)$ is reduced by the antioxidant compound $(\mathrm{ArOH})$ to the ferrous form ( $\mathrm{Fe}(\mathrm{TPTZ})_{2}^{3+}$ ) releasing the oxidised form of the antioxidant compound $\left(\mathrm{ArOH}^{+}\right)$.

Absorbance measurements were recorded on a U-2800 Spectrophotometer Digilab Hitachi at $25^{\circ} \mathrm{C} . \mathrm{GLP} 22$ Crison pH-meter was used for buffer preparation. Briefly, $3 \mathrm{ml}$ of FRAP reactive: (10:1:1) acetate buffer (300 mM, pH 3, 6), TPTZ (10Mm in $\mathrm{HCl} 40 \mathrm{Mm})$ and $\mathrm{FeCl}_{3} \cdot 6 \mathrm{H}_{2} \mathrm{O}(20 \mathrm{mM}) ; 100 \mu$ l problem solution, and $300 \mu \mathrm{l}$ mili Q water. Although the method proposed to determine the absorbance at 4 min, we monitored the reaction until finished for each compound at $593 \mathrm{~nm}$. Data were translated into FRAP value $(\mu \mathrm{M})$ using an aqueous solution of $\mathrm{FeSO}_{4} \cdot 7 \mathrm{H}_{2} \mathrm{O}$ in the range of $0-1000 \mu \mathrm{M}[13]$.

\section{Results and Discussion}

\subsection{Antioxidant Activity Values}

For comparative purposes, Table 1 shows the antioxidant activity values for each compound obtained by ORAC and FRAP methods at equimolar concentrations. Uric acid presents a stable and a well known concentration in plasma ranging between $0.277-0.416 \mathrm{mM}$. Hence, we selected $0.416 \mathrm{mM}$ as a reference concentration to compare the compounds under study in terms of antioxidant activity.

Different mechanisms are involved in both antioxidant methods. Thus, ORAC method is based on the evaluation of the oxidative damage produced by peroxyl radical against fluorescein, as a consequence fluorescence decrease. The peroxyl radical is generated by thermic decomposition of AAPH. After that, the radical react with the fluorescein and the decrease of fluorescence signal is measured. In the presence of an antioxidant compound $(\mathrm{ArOH})$, the reaction with the fluorescein is delayed and a phase of retard is produced. The higher the efficacy of the antioxidant compound for scavenging free radicals, the lower the decrease of the florescein intensity, that is, the area under the curve (intensity emission/time) would be higher [14]:

$$
\mathrm{ROO}+\mathrm{ArOH} \rightarrow \mathrm{ArO}+\mathrm{ROOH}
$$

Equation (2) shows the HAT mechanism of ORAC method, as follows: an antioxidant compound (ArOH) in presence of a peroxyl radical (ROO) transfer a hydrogen atom and transform it in other compounds less harmful $(\mathrm{ROOH})$.

\begin{tabular}{|c|c|c|}
\hline \multirow[b]{2}{*}{ Compounds } & \multicolumn{2}{|c|}{ Antioxidant activity values } \\
\hline & $\begin{array}{c}\text { ORAC } \\
\text { ( } \mu \mathrm{m} \text { Trolox/mmolcompund) }\end{array}$ & $\begin{array}{c}\text { FRAP } \\
\text { ( } \mu \mathrm{M} \mathrm{Fe}^{2+} / \text { mmolcompund) }\end{array}$ \\
\hline Uric acid & $7015.3 \pm 498.5$ & $854.4 \pm 79.7$ \\
\hline Ascorbic acid & $22685.8 \pm 1257.1$ & $782.7 \pm 21.7$ \\
\hline Protocatechuic acid & $20582.9 \pm 688.4$ & $2315.4 \pm 53.1$ \\
\hline 3,4-dihydroxyphenylacetic acid & $10048.1 \pm 240.4$ & $4303.4 \pm 57.3$ \\
\hline 3,4-dihydroxyphenylpropionic acid & $23093.9 \pm 472.1$ & $3539.6 \pm 159.4$ \\
\hline 3-hydroxyphenylacetic acid & $1844.2 \pm 179.8$ & $543.8 \pm 14.4$ \\
\hline
\end{tabular}

Table 1. Antioxidant activity values of standard compounds determined by ORAC and FRAP methods.

${ }^{*}$ Compounds concentration $0.416 \mathrm{mM}$. 
On the other hand, FRAP method is based on SET mechanism. The reaction measure an oxide-reduction of ferric-tripyridyltriazine ( $\mathrm{Fe}^{\mathrm{III}}$-TPTZ) complex to the ferrous $\left(\mathrm{Fe}^{\mathrm{II}}\right)$ form in the presence of an antioxidant (1). An intense blue color with an absorption maximum at $593 \mathrm{~nm}$ is measured [13].

In summary, it could be established that ascorbic acid and 3,4-dihydroxyphenylpropionic acid, whose activity rank for the higher values when determined by ORAC methods, work by a HAT mechanism. Therefore, those compounds have higher antioxidant activity in the biological systems in which HAT mechanism is prevalent. Thus, biological pH is close to that of ORAC method. It is relevant the case of ascorbic acid, since it is the principal exogenous antioxidant present in many foods.

On the other hand, antioxidants which rank for the higher reactivity by FRAP method, such as 3,4-dihydroxyphenyacetic acid, may involve a SET mechanism. Protocatechuic acid, uric acid and 3-hydroxyphenylacetic acid displays a similar ranking by both methods. Therefore, they could act equally by HAT or SET.

\subsection{Kinetic Study}

The velocity of reaction (v) is relevant from a physiological point because it is important that antioxidants scavenge the radicals before they react with the target and produce oxidative damage.

ORAC method involves radicals' production and its reaction with the target molecule (fluorescein). Fluorescein (FL) is always in excess, that is, its concentration is higher than the radical. Although the radical concentration decreases because of the antioxidant, fluorescein will not significantly be affected. Therefore, the experimentally observed rate constant, $\mathrm{k}_{\mathrm{obs}}$, remains constant.

On the other hand, FRAP method measure the absorbance of the Fe ${ }^{\mathrm{II}-} \mathrm{TPTZ}$ complex with the ligand $(\mathrm{L})$. The antioxidant present in the medium drive to the formation of $\mathrm{Fe}^{\mathrm{II}}$ by reduction of $\mathrm{Fe}^{\mathrm{III}}$. Since the $\mathrm{Fe}^{\mathrm{III}}$-TPTZ complex is in excess the velocity of reaction will be determinate by the $\mathrm{Fe}^{\mathrm{II}}$ formed, which will depend on the antioxidant concentration. Thus, FRAP method allow to assess the velocity of the reaction taking place because it is a direct measure, that is, the reaction velocity is determined by the concentration of the $\mathrm{Fe}^{\mathrm{II}}$ complex formed with the ligand and the concentration of the antioxidant.

The reaction rate equation can be expressed as:

$$
\mathrm{v}=\mathrm{k}\left(\mathrm{Fe}^{\mathrm{II}} \mathrm{L}\right)(\mathrm{ArOH})=\mathrm{k}_{\mathrm{obs}}(\mathrm{ArOH})
$$

Here $k$ is the second order rate constant and $\mathrm{k}_{\mathrm{obs}}=\mathrm{k}\left(\mathrm{Fe}^{\mathrm{II}} \mathrm{L}\right)$. One can write:

$$
\mathrm{v}=-\mathrm{d}(\mathrm{ArOH}) / \mathrm{dt}=\mathrm{k}_{\mathrm{obs}}(\mathrm{ArOH})
$$

And integrating are obtains:

$$
\mathrm{Ln}(\mathrm{ArOH})_{\mathrm{t}}=\mathrm{Ln}(\mathrm{ArOH})_{0}-\mathrm{k}_{\mathrm{obs}^{*}} \mathrm{t}
$$

Here $(\mathrm{ArOH})_{0}$ and $(\mathrm{ArOH})_{\mathrm{t}}$ are the antioxidant concentration at $\mathrm{t}=0$ and $\mathrm{t}=$ certain time, respectively.

Absorbance is proportional to the $\mathrm{Fe}^{\mathrm{II}}$-TPTZ concentration. The final absorbance, Af, is proportional to the final Fe ${ }^{\mathrm{II}} \mathrm{TPTZ}$ concentration, that is, to the initial antioxidant concentration. Absorbance at a certain time, At, is proportional to the $\mathrm{Fe}^{\mathrm{II}}$-TPTZ concentration formed. Therefore, Af-At is proportional to the remaining antioxidant concentration at certain time. Thus, Equation (5) can be explained as:

$$
\operatorname{Ln}(\text { Af }- \text { At })=a-K_{\text {obs }^{*}} \mathrm{t}
$$

Here $a$ is a constant proportional to the initial antioxidant concentration.

Information about the antioxidant activity of the different antioxidants can be obtained from the calculated $\mathrm{k}_{\mathrm{obs}}$ values (Figure 1, Table 2). It is worth noting that the absorbance values were registered during a $480 \mathrm{~s}$ time interval in order to make sure that the oxidation reaction is finished for all the antioxidants investigated (see Figure 2 for uric acid and3,4-dihydroxyphenylacetic acid).

\subsection{Evaluation of Synergism: Antioxidant Activity Values and Kinetic Criteria}

In order to evaluate the synergism between different compounds, the antioxidant activity (ORAC and FRAP methods) of different combinations of selected endogenous (uric acid) and exogenous (ascorbic acid and phenolic metabolites) compounds was determined. 


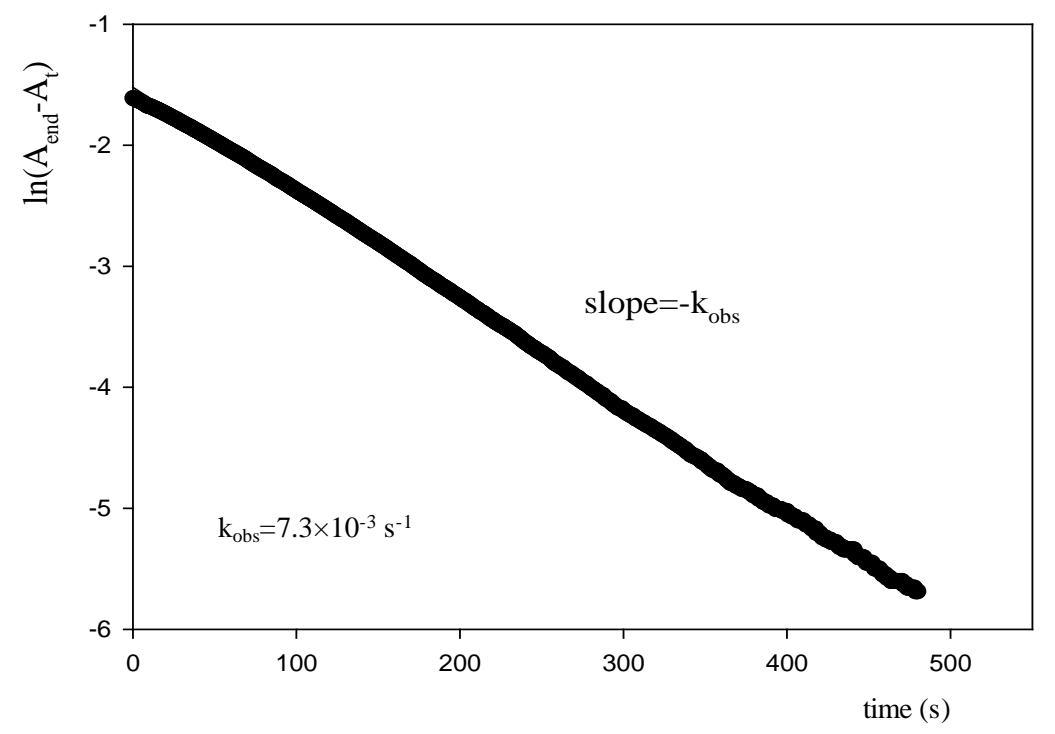

Figure 1. Slope for observed rate constant of compounds.

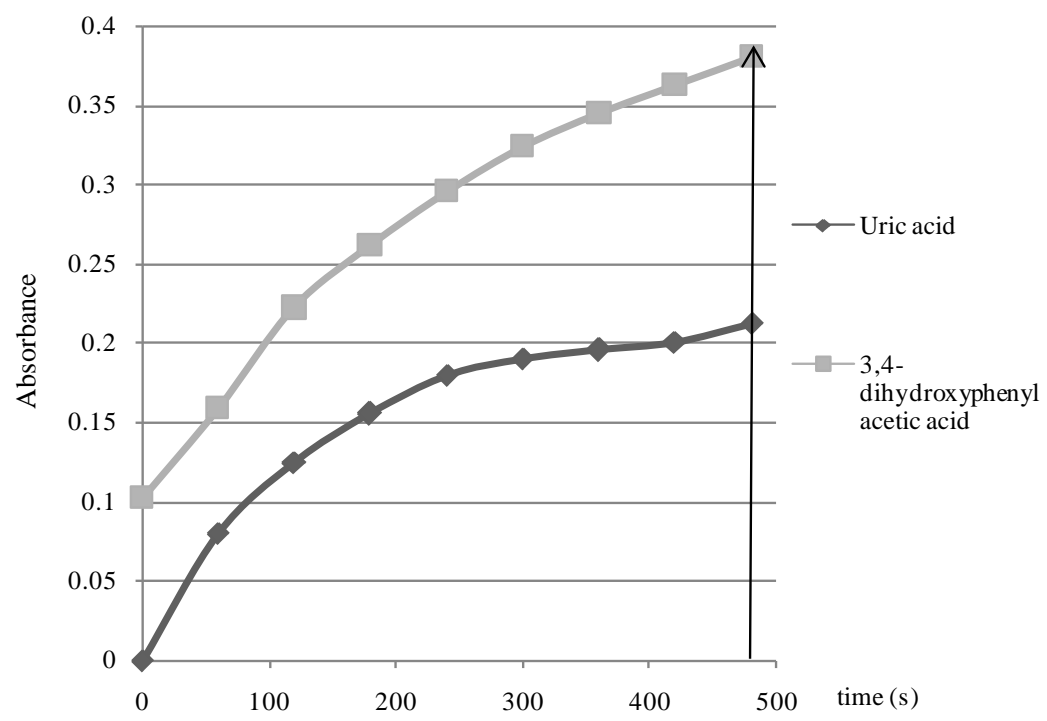

Figure 2. Absorbance values for uric acid and 3,4-dihydroxyphenylacetic acid determined by FRAP method.

Table 2. Observed rate constant valuefor the different compounds.

\begin{tabular}{lc}
\hline \multicolumn{1}{c}{ Compounds $^{*}$} & $\begin{array}{c}\text { Observed rate constant } \\
\left(\mathrm{Kobs} / \mathrm{s}^{-1}\right)\end{array}$ \\
\hline Uric acid & 0.007 \\
Ascorbic acid & 0.007 \\
Protocatechuic acid & 0.012 \\
3,4-dihydroxyphenylacetic acid & 0.013 \\
3,4-dihydroxyphenylpropionic acid & 0.013 \\
\hline
\end{tabular}

*Compounds concentration $0.416 \mathrm{mM}$.

Table 3 and Table 4 show experimental and theoretical antioxidant activity values for different combinations of compounds determined by ORAC and FRAP, respectively. Experimental value was obtained by assessing a 
Table 3. Antioxidant activity values for the combination of the different compounds determined by ORAC method.

\begin{tabular}{lccc}
\hline \multicolumn{1}{c}{ Compounds* } & \multicolumn{2}{c}{ Antioxidant activity values by ORAC $(\mu \mathrm{M}$ trolox $)$} \\
\cline { 2 - 4 } & Experimental measurements & Theoretical measurements & Synergism \\
\hline Uric acid + ascorbic acid & $4221.8 \pm 363.3^{\mathrm{a}}$ & $12355.6 \pm 459.3^{\mathrm{b}}$ & $\mathrm{NO}$ \\
uric acid + Protocatechuic acid & $12150.0 \pm 410.1$ & $11480.8 \pm 288.7$ & $\mathrm{X}$ \\
Uric acid + 3,4-dihydroxyphenylacetic acid & $6636.6 \pm 600.2$ & $7098.4 \pm 178.9$ & $\mathrm{X}$ \\
Uric acid + 3,4-dihydroxyphenylpropionic acid & $9888.0 \pm 916.4$ & $12529.4 \pm 207.6$ & $\mathrm{X}$ \\
Uric acid + 3-hydroxyphenylacetic acid & $1009.7 \pm 372.7^{\mathrm{a}}$ & $3685.3 \pm 174.6^{\mathrm{b}}$ & $\mathrm{NO}$ \\
Ascorbic acid + Protocatechuic acid & $11615.0 \pm 3174.0$ & $17999.8 \pm 486.8$ & $\mathrm{X}$ \\
Ascorbic acid + 3,4-dihydroxyphenylacetic acid & $16773.1 \pm 836.7^{\mathrm{a}}$ & $13617.3 \pm 430.8^{\mathrm{b}}$ & YES \\
Ascorbic acid + 3,4-dihydroxyphenylpropionic acid & $1050.6 \pm 75.6^{\mathrm{a}}$ & $19048.3 \pm 443.5^{\mathrm{b}}$ & $\mathrm{NO}$ \\
Ascorbic acid + 3-hydroxyphenylacetic acid & $1973.5 \pm 172.9^{\mathrm{a}}$ & $10204.3 \pm 429.1^{\mathrm{b}}$ & $\mathrm{NO}$ \\
Uric acid + ascorbic acid + protocatechuic acid & $31575.5 \pm 5456.7^{\mathrm{a}}$ & $20918.2 \pm 477.2^{\mathrm{b}}$ & YES \\
Uric acid + Ascorbic acid + 3,4-dihydroxyphenylacetic acid & $6662.3 \pm 109.1^{\mathrm{a}}$ & $16535.6 \pm 428.6^{\mathrm{b}}$ & $\mathrm{NO}$ \\
Uric acid + Ascorbic acid + 3,4-dihydroxyphenylpropionic acid & $10919.6 \pm 1618.8^{\mathrm{a}}$ & $21966.6 \pm 439.5^{\mathrm{b}}$ & $\mathrm{NO}$ \\
Uric acid + Ascorbic acid + 3-hydroxyphenylacetic acid & $7495.6 \pm 347.1^{\mathrm{a}}$ & $13122.6 \pm 427.1^{\mathrm{b}}$ & $\mathrm{NO}$ \\
\hline
\end{tabular}

*Compounds concentration $0.416 \mathrm{mM}$. ${ }^{\text {ab }}$ superscript letters in the same column indicate significant differences (p < 0.05); NO: experimental measurement was significantly lesser than theoretical measurement; X: no significant differences between experimental and theoretical measurementswere observed; YES: experimental measurements was significantly higher than theoretical measurements.

Table 4. Antioxidant activity values for the combination of the different compounds determined by FRAP method.

\begin{tabular}{|c|c|c|c|}
\hline \multirow{2}{*}{ Compounds $^{*}$} & \multicolumn{3}{|c|}{ Antioxidant activity values by FRAP $\left(\mu \mathrm{M} \mathrm{Fe}^{2+}\right)$} \\
\hline & Experimental measurements & Theoretical measurements & Sinergism \\
\hline Uric acid+ascorbic acid & $2210.6 \pm 66.3^{\mathrm{a}}$ & $681.1 \pm 28.1^{b}$ & YES \\
\hline uric acid+ Protocatechuic acid & $1404.6 \pm 4.4$ & $1318.7 \pm 32.5$ & $\mathrm{X}$ \\
\hline Uric acid+3,4-dihydroxyphenylacetic acid & $1641.8 \pm 25.3$ & $2145.7 \pm 33.4$ & $\mathrm{X}$ \\
\hline Uric acid+3,4-dihydroxyphenylpropionic acid & $4491.8 \pm 66.3^{\mathrm{a}}$ & $1827.9 \pm 60.5^{b}$ & YES \\
\hline Uric acid+3-hydroxyphenylacetic acid & $46.6 \pm 1.0$ & $581.6 \pm 27.5$ & NO \\
\hline Ascorbic acid+Protocatechuic acid & $1014.0 \pm 8.8$ & $1288.8 \pm 19.5$ & $\mathrm{X}$ \\
\hline Ascorbic acid+3,4-dihydroxyphenylacetic acid & $839.0 \pm 8.8^{\mathrm{a}}$ & $2115.8 \pm 22.5^{b}$ & NO \\
\hline Ascorbic acid+3,4-dihydroxyphenylpropionic acid & $304.4 \pm 22.1^{\mathrm{a}}$ & $1798.1 \pm 54.6^{\mathrm{b}}$ & NO \\
\hline Ascorbic acid+3-hydroxyphenylacetic acid & $51.9 \pm 11.5^{\mathrm{a}}$ & $551.8 \pm 8.8^{b}$ & NO \\
\hline Uric acid+ascorbic acid+protocatechuic acid & $1402.2 \pm 33.2$ & $1647.9 \pm 30.8$ & $\mathrm{X}$ \\
\hline Uric acid+Ascorbic acid+3,4-dihydroxyphenylacetic acid & $4913.7 \pm 88.4^{\mathrm{a}}$ & $2471.2 \pm 32.5^{b}$ & YES \\
\hline Uric acid+Ascorbic acid+3,4-dihydroxyphenylpropionic acid & $710.6 \pm 22.1^{\mathrm{a}}$ & $2153.5 \pm 56.4^{b}$ & NO \\
\hline Uric acid+Ascorbic acid+3-hydroxyphenylacetic acid & $198.4 \pm 16.9^{\mathrm{a}}$ & $907.2 \pm 26.3^{b}$ & NO \\
\hline
\end{tabular}

${ }^{*}$ Compounds concentration $0.416 \mathrm{mM}$. ${ }^{\mathrm{ab}}$ superscript letters in the same column indicate significant differences (p < 0.05); NO: experimental measurement was significantly lesser than theoretical measurement; X: no significant differences between experimental and theoretical measurements were observed; YES: experimental measurements was significantly higher than theoretical measurements.

mix of compounds. While theoretical value was calculated by summing the values obtained for each compound individually.

It was observed a synergism effect in the combination of ascorbic acid with 3,4-dihydroxyphenylacetic acid, and ascorbic acid with uric acid and protocatechuic acid rising 1.2 and 1.5 fold their theoretical values respectively, when they were measured by ORAC. According to the FRAP method, synergic effect was shown for different combinations of compounds: uric acid with ascorbic acid; uric acid with 3,4-dihydroxyphenylpropionic, and uric acid with ascorbic acid and 3,4-dihydroxyphenylacetic acid, increasing 3.23, 2.45 and 2 folds their theoretical values, respectively. 
Table 5. Observed rate constant values for the different compounds.

\begin{tabular}{lc}
\hline \multicolumn{1}{c}{ Compounds } & $\begin{array}{c}\text { Observed rate constant } \\
\left(\mathrm{Kobs} / \mathrm{s}^{-1}\right)\end{array}$ \\
\hline Protocatechuic acid + uric acid & 0.012 \\
Protocatechuic acid + ascorbic acid & 0.012 \\
Protocatechuic acid + uric acid + ascorbic acid & 0.012 \\
3,4-dihydroxyphenylacetic acid + uric acid & 0.005 \\
3,4-dihydroxyphenylacetic acid + ascorbic acid & 0.190 \\
3,4-dihydroxyphenylacetic acid + uric acid + ascorbic acid & 0.004 \\
3,4-dihydroxyphenylpropionic acid + uric acid & 0.010 \\
3,4-dihydroxyphenylpropionic acid + ascorbic acid & 0.010 \\
3,4-dihydroxyphenylpropionic acid + uric acid + ascorbic acid & 0.010 \\
\hline
\end{tabular}

Finally synergism was evaluated in kinetic terms and the constants of the different combinations were determined (Table 5). As can be seen the combination of ascorbic acid with 3,4-dihydroxyphenylacetic acid presented the highest rate constant, showing a potent synergism effect being relevant for biological perspective. Hence, this combination includes both aspects antioxidant activity itself and a marked reaction rate.

3,4-Dihydroxyphenylacetic acid is a metabolite produced by the colonic microbiota [15] [16], from flavonoids (anthocyanin and quercetin). This metabolite proved to preserve neuron cell from death due to oxidative stress [16]. Additionally, this metabolite is a powerful inhibitor of $\mathrm{N}^{\varepsilon}$-carboxymethyl lysine and $\mathrm{N}^{\varepsilon}$-carboxymethyl lysine-histone H1 adduct formation and ADP-ribose histone H1glycation [17]. Furthermore, 3,4-dihydroxyphenylacetic acid has been demonstrated to act as an anti-inflammatory agent, probably contributing to the chemopreventive potential of phenolic metabolites in the gut [18].

\section{Conclusion}

In conclusion, we have established that there is synergism effect between exogenous antioxidants (ascorbic acid and 3,4-dihydroxyphenylacetic acid) and between endogenous and exogenous antioxidants (ascorbic acid, uric acid and protocatechuic acid; uric acid and ascorbic acid; uric acid and 3,4-dihydroxyphenylpropionic acid, and uric acid, ascorbic acid and 3,4-dihydroxyphenylacetic acid). Additionally, the combination of ascorbic acid with the colonic microbiota metabolite 3,4-dihydroxyphenylacetic acid showed a potent synergism effect relevant for biological perspective due to its market reaction rate.

\section{Acknowledgements}

The authors are grateful to the Spanish Ministry of Economy and Competitiveness for financial funding (AGL2010-22152-C03-01).

\section{References}

[1] Borbalán, A., Zorro, L., Guillén, D. and García-Barroso, C. (2003) Study of the Polyphenol Content of Red and White Wine Grape Varieties by Liquid Chromatography-Mass Spectrometry and Its Relationship to Antioxidant Power. Journal of Chromatography, 1012, 31-38. http://dx.doi.org/10.1016/S0021-9673(03)01187-7

[2] De Beer, D., Joubert, E., Gelderblom, W. and Manley M. (2003) Antioxidant Activity of South African Red Wines and White Cultivar Wines: Free Radical Scavenging. Journal of Agricultural and Food Chemistry, 51, 902-909. http://dx.doi.org/10.1021/jf0260110

[3] Sánchez-Moreno, C., Cao, G., Ou, B. and Prior, R.L. (2003) Anthocyanin and Proanthocyanidin Content in Selected White and Red Wines. Oxygen Radical Absorbance Capacity Comparison with Nontraditional Wines Obtained from Highbush Blueberry. Journal of Agricultural and Food Chemistry, 51, 4889-4896. http://dx.doi.org/10.1021/jf030081t

[4] Fernández-Pachón, M.S., Villaño, D., García-Parrilla, M.C. and Troncoso, A.M. (2004). Antioxidant Activity of Wines and Relation with Their Polyphenolic Composition. Analytica Chemica Acta, 513, 113-118.

[5] Prior, R., Xianli, W. and Schaich, K. (2005) Standarized Methods for the Determination of Antioxidant Capacity and Phenolics in Foods and Dietary Supplements. Journal of Agriculture and Food Chemistry, 53, 4290-4302. 
http://dx.doi.org/10.1021/jf0502698

[6] Landrault, N., Poucheret, P., Ravel, P., Gasc, F., Cros, G. and Teissedre, P.L. (2001) Antioxidant Capacities and Phenolics Levels of French Wines from Different Varieties and Vintages. Journal of Agricultural and Food Chemistry, 49, 3341-3348. http://dx.doi.org/10.1021/jf010128f

[7] González-Paramás, A.M., Esteban-Ruano, S., Santos-Buelga, C., De Pascual-Reresa, S. and Rivas-Gonzálo, J.C. (2004) Flavanol Content and Antioxidant Activity in Winery Byproducts. Journal of Agricultural and Food Chemistry, 52, 234-238. http://dx.doi.org/10.1021/jf0348727

[8] Fernandez-Pachón, M.S., Villaño, D., Troncoso, A.M. and García-Parrilla, M.C. (2006) Determination of the Phenolic Composition of Sherry and Table White Wines by Liquid Chromatography and Their Relation with Antioxidant Activity. Analytica Chemica Acta, 563, 101-108.

[9] Aaby, K., Ekeberg, D. and Skrede, G. (2007) Characterization of Phenolic Compounds in Strawberry (Fragaria $x$ ananassa) Fruits by Different HPLC Detectors and Contribution of Individual Compounds to Total Antioxidant Capacity. Journal of Agricultural and Food Chemistry, 55, 4395-4406. http://dx.doi.org/10.1021/jf0702592

[10] Aaby, K., Wrolstad, R.E., Ekeberg, D. and Skrede, G. (2007) Polyphenol Composition and Antioxidant Activity in Strawberry Purees; Impact of Achene Level and Storage. Journal of Agricultural and Food Chemistry, 55, 5156-5166. http://dx.doi.org/10.1021/jf070467u

[11] Kinnula, V.L. and Crapo, J.D. (2003) Superoxide dismutases in the Lung and Human Lung Diseases. American Journal of Respiratory and Critical Care Medicine, 167, 1600-1619.

[12] Del Rio, D., Rodriguez-Mateos, A., Spencer, J.P.E., Tognolini, M., Borges, G. and Crozier, A. (2013) Dietary (Poly)Phenolics in Human Health: Structures, Bioavailability, and Evidence of Protective Effects Against Chronic Diseases. Antioxidants and Redox Signaling, 18, 1818-1892. http://dx.doi.org/10.1089/ars.2012.4581

[13] Benzie, I.F.F. and Strain, J.J. (1996) The Ferric Reducing Ability of Plasma as a Measure of “Antioxidant Power”: The FRAP Assay. Analytical Biochemistry, 239, 70-76. http://dx.doi.org/10.1006/abio.1996.0292

[14] Prior, R.L. and Cao, G. (1999) In Vivo Total Antioxidant Capacity: Comparison of Different Analytical Methods. Free Radical Biology \& Medicine, 27, 1173-1181. http://dx.doi.org/10.1016/S0891-5849(99)00203-8

[15] Jaganath, I.B., Mullen, W., Lean, M.E., Edwards, C.A., and Crozier, A. (2009) In vitro Catabolism of Rutin by Human Fecal Bacteria and the Antioxidant Capacity of Its Catabolites. Free Radical Biology \& Medicine, 47, 1180-1189. http://dx.doi.org/10.1016/j.freeradbiomed.2009.07.031

[16] Williamson, G. and Clifford, M.N. (2010) Colonic Metabolites of Berry Polyphenols: The Missing Link to Biological Activity? British Journal of Nutrition, 104, S48-S66. http://dx.doi.org/10.1017/S0007114510003946

[17] Pashikanti, S., de Alba, D.R., Boissonneault, G.A. and Cervantes-Laurean, D. (2010) Rutin Metabolites: Novel Inhibitors of Nonoxidative Advanced Glycation End Products. Free Radical Biology \& Medicine, 48, 656-663.

[18] Miene, C., Weise, A. and Glei M. (2011) Impact of Polyphenol Metabolites Produced by Colonic Microbiota on Expression of COX-2 and GSTT2 in Human Colon Cells (LT97). Nutrition and Cancer, 63, 653-662.

http://dx.doi.org/10.1016/j.freeradbiomed.2009.11.019 